\title{
Number of prime divisors in a product of consecutive integers
}

\author{
by \\ Shanta Laishram and T. N. Shorey (Mumbai) \\ Dedicated to Professor Robert Tijdeman on his 60th birthday
}

1. Introduction. Let $n$ and $k \geq 2$ be positive integers. For a pair $(n, k)$ and a positive integer $h$, we write $[n, k, h]$ for the set of all pairs $(n, k), \ldots,(n+h-1, k)$ and we set $[n, k]=[n, k, 1]=\{(n, k)\}$. Further we put

$$
\Delta:=\Delta(n, k)=n(n+1) \cdots(n+k-1) .
$$

We use the above notation for any positive integer $x$ in place of $n$ and we write

$$
\Delta^{\prime}:=\Delta(x-k+1, k)=x(x-1) \cdots(x-k+1) .
$$

For an integer $\nu>1$, we denote by $\omega(\nu)$ and $P(\nu)$ the number of distinct prime divisors of $\nu$ and the greatest prime factor of $\nu$, respectively, and let $\omega(1)=0, P(1)=1$. Also we denote by $W\left(\Delta^{\prime}\right)$ the number of terms in $\Delta^{\prime}$ divisible by a prime greater than $k$. Let $p_{i}$ denote the $i$ th prime number. Thus $p_{1}=2, p_{2}=3, \ldots$ We shall always write $p$ for a prime number.

Since $\Delta$ is divisible by $k$ !, we observe that

$$
\omega(\Delta) \geq \pi(k)
$$

where $\pi(k)$ denotes the number of primes not exceeding $k$. A well known theorem of Sylvester [12] states that

$$
\omega(\Delta)>\pi(k) \text { if } n>k .
$$

This means that a product of $k$ consecutive integers each exceeding $k$ is divisible by a prime greater than $k$. It is clear that the assumption $n>k$ in (1) is necessary since $\omega(\Delta(1, k))=\pi(k)$. Another proof of Sylvester's theorem was given by Erdös [5]. Saradha and Shorey [10, Corollary 3] extended the

2000 Mathematics Subject Classification: 11B25, 11N13.

Research of S. Laishram partially supported by TIFR Endowment Fund. 
proof of Erdős to sharpen (1) as

$$
\omega(\Delta) \geq \pi(k)+\left[\frac{1}{3} \pi(k)\right]+2 \quad \text { if } n>k>2
$$

unless $(n, k) \in S_{1}$, where $S_{1}$ is the union of the sets

$$
\left\{\begin{array}{l}
{[4,3],[6,3,3],[16,3],[6,4],[6,5,4],[12,5],[14,5,3],[23,5,2],} \\
{[7,6,2],[15,6],[8,7,3],[12,7],[14,7,2],[24,7],[9,8],[14,8],} \\
{[14,13,3],[18,13],[20,13,2],[24,13],[15,14],[20,14],[20,17] .}
\end{array}\right.
$$

We define

$$
\delta:=\delta(k)= \begin{cases}3 & \text { if } k=2 \\ 2 & \text { if } 3 \leq k \leq 6 \\ 1 & \text { if } 7 \leq k \leq 16 \\ 0 & \text { otherwise }\end{cases}
$$

so that

$$
\left[\frac{3}{4} \pi(k)\right]-1+\delta(k) \geq\left[\frac{1}{3} \pi(k)\right]+2 .
$$

We observe that

$$
\omega(\Delta)=\pi(2 k) \quad \text { if } n=k+1 .
$$

Therefore, the constant $1 / 3$ in (2) cannot be replaced by a number larger than 1 . In the present paper, we replace $1 / 3$ by $3 / 4$. But in that case, it is necessary to enlarge the set $S_{1}$ of exceptions. We derive from Lemma 1(i), (ii) that the inequality

$$
\pi(k)+\left[\frac{3}{4} \pi(k)\right]-1+\delta(k)>\pi(2 k)
$$

implies $k \leq 2697$. Further we check that the values of $k \leq 2697$ satisfying (5) are given by $k$ in $\left\{\begin{array}{l}2,3,5,6,7,8,13,14,19,20,47,48,73,74,83,89,107,108,109,110,111,112, \\ 113,114,115,116,173,199,200,277,278,281,282,283,284,285,293 .\end{array}\right.$

Therefore we see from (4) that it is necessary to include at least the exceptions given by $n=k+1$ for the above values of $k$. We prove

Theorem 1. Let $n>k \geq 3$. Then

$$
\omega(\Delta) \geq \pi(k)+\left[\frac{3}{4} \pi(k)\right]-1+\delta(k)
$$

unless

$$
(n, k) \in S_{1} \cup S_{2}
$$


where $S_{1}$ is given by (3) and $S_{2}$ is the union of the sets

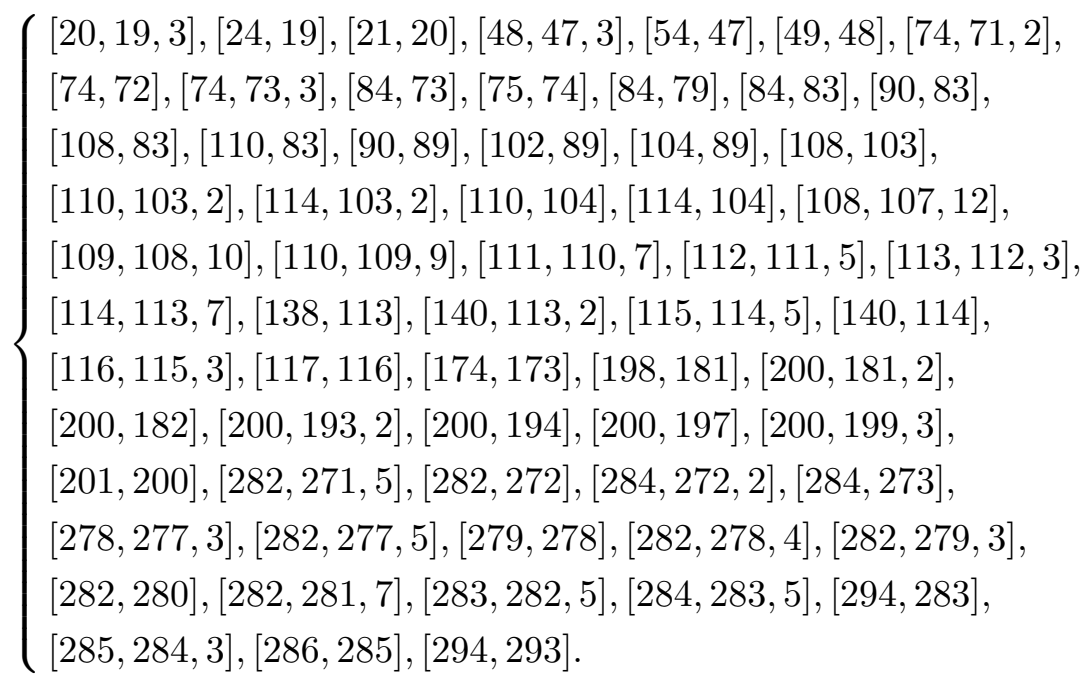

Catalan [1] conjectured in 1844 that 8 and 9 are the only perfect powers that differ by 1 . Tijdeman [13] proved in 1976 that there are only finitely many perfect powers that differ by 1 . A complete proof of Catalan's conjecture has been established recently by Mihăilescu [6]. This implies that exceptions to (6) when $k=2$ and $n \neq 8$ are given by Mersenne and Fermat primes.

It follows from Theorem 1 that (6) holds for $k>293$. Further we observe that the largest value of $k$ in $S_{1} \cup S_{2}$ coincides with the largest value of $k$ satisfying (5). Next we check that the exceptions given in Theorem 1 are necessary. It is clear that Theorem 1 includes the result of Saradha and Shorey stated above. In fact both the results are identical in $3 \leq k \leq 18$. Thus it suffices to prove Theorem 1 for $k \geq 19$. We derive two results from Theorem 1, the first one with no exception and the second one with only two exceptions.

If $k+1$ is prime and $2 k+1$ is composite, then we observe by writing

$$
\Delta(k+2, k)=\Delta(k+1, k) \frac{2 k+1}{k+1}
$$

that

$$
\omega(\Delta(k+2, k))=\pi(2 k)-1 .
$$

We notice that there are infinitely many $k$ for which $k+1$ is prime and $2 k+1$ is composite. Therefore (8) is valid for infinitely many $k$. Thus an inequality sharper than $\omega(\Delta) \geq \pi(2 k)-1$ for $n>k$ is not valid. Further we observe that

$$
\omega(\Delta(n, k)) \geq \pi(2 k)-1 \quad \text { if } n=k+3, k+4, k+5 .
$$


It is difficult to show the above inequality when $n=k+6$ since we cannot exclude the possibility that $N, N+2$ are primes and $2 N-5,2 N-3$ are composites for $N=k+3$. For $N \leq 10^{9}$, there are 2487118 values of $N$ with $N, N+2$ primes and $2 N-5,2 N-3$ composites. If there are infinitely many such $N$, then $\omega(\Delta(N+3, N-3))=\pi(2 N-6)-2$ for infinitely many $N$. We conjecture that there are infinitely many twin primes $N, N+2$ such that $2 N-5,2 N-3$ are composites.

We observe from (4), (8) and (9) that (10) is valid for all exceptions $(n, k)$ with $k+1 \leq n \leq k+5$ in Theorem 1 . Further we check that other exceptions in Theorem 1 satisfy $\omega(\Delta) \geq \pi(2 k)-1$ implying (10) again. Therefore we deduce from Theorem 1 the following result.

COROllary 1. Let $n>k$. Then

$$
\omega(\Delta) \geq \min \left(\pi(k)+\left[\frac{3}{4} \pi(k)\right]-1+\delta(k), \pi(2 k)-1\right) .
$$

Corollary 2. Let $n>k$. Then

$$
\omega(\Delta) \geq \pi(k)+\left[\frac{2}{3} \pi(k)\right]-1
$$

unless

$$
(n, k) \in\{(114,109),(114,113)\} .
$$

For the proof of Corollary 2, we may assume that $(n, k) \in S_{1} \cup S_{2}$ by Theorem 1. By Corollary 1, we may suppose that

$$
\pi(2 k)-1<\pi(k)+\left[\frac{2}{3} \pi(k)\right]-1,
$$

which implies that $k=5,13,14,19,20,23,47,48,73,74,109,110,111,113$, 114. Now we check (11) for all pairs $(n, k)$ given above other than (12). The estimate (2) has been applied in [7] and [11]. Further Theorem 1 and Corollary 1 have also been applied in [7].

The constant $3 / 4$ in Theorem 1 can be replaced by a number close to 1 if $n>\frac{17}{12} k$.

Theorem 2. Let $(n, k) \neq(6,4)$. Then

$$
\omega(\Delta) \geq \pi(2 k) \quad \text { if } n>\frac{17}{12} k .
$$

We observe that $\frac{17}{12} k$ in Theorem 2 is optimal since $\omega(\Delta(34,24))=$ $\pi(48)-1$. Also the assumption $(n, k) \neq(6,4)$ is necessary since $\omega(\Delta(6,4))=$ $\pi(8)-1$. Some of the pairs $(n, k)$ for which $\omega(\Delta) \geq \pi(2 k)$ is not valid are 
as follows:

$$
\omega(\Delta)= \begin{cases}\pi(2 k)-1 & \text { if }(n, k)=(6,4),(34,24),(33,25),(80,57), \\ \pi(2 k)-2 & \text { if }(n, k)=(74,57),(284,252),(3943,3880), \\ \pi(2 k)-3 & \text { if }(n, k)=(3936,3879),(3924,3880),(3939,3880), \\ \pi(2 k)-4 & \text { if }(n, k)=(1304,1239),(1308,1241),(3932,3879), \\ \pi(2 k)-5 & \text { if }(n, k)=(3932,3880),(3932,3881),(3932,3882) .\end{cases}
$$

Now we give a formula from which the above calculation have been derived. Let $n=k+r$ with $0<r \leq k$. Observing that the prime factors of $\Delta$ are the $p$ 's satisfying either $p \leq k+(r-1) / 2$ or $k+r \leq p \leq 2 k+r-1$, we obtain

$$
\omega(\Delta(n, k))=\pi(2 k)+F(k, r)
$$

where

$$
F(k, r)=\pi(2 k+r-1)-\pi(2 k)-\left(\pi(k+r-1)-\pi\left(k+\frac{r-1}{2}\right)\right) .
$$

It is also possible to replace $3 / 4$ in Theorem 1 by a number close to 1 if $n>k$ and $k$ is sufficiently large. Let $\varepsilon>0$ and $n>k$. Then there exists $k_{0}$ depending only on $\varepsilon$ such that for $k \geq k_{0}$, we have

$$
\omega(\Delta(n, k)) \geq(2-\varepsilon) \pi(k) .
$$

For a proof, we may suppose that $n \leq \frac{17}{12} k$ by Theorem 2 . Further

$$
\omega(\Delta) \geq \pi(n+k-1)-\pi(n-1)+\pi(k) .
$$

Now the assertion (14) follows from the Prime Number Theorem.

Saradha and Shorey derived (2) from a more general result [10, Theorem $\left.3^{\prime}\right]$ :

Theorem A. Let $x \geq 2 k$ and $f_{1}<f_{2}<\cdots<f_{\mu}$ be integers in $[0, k)$. Assume that

$$
P\left(\left(x-f_{1}\right) \cdots\left(x-f_{\mu}\right)\right) \leq k
$$

Then

$$
\mu \leq k-\left[\frac{1}{3} \pi(k)\right]-2
$$

unless $(x, k) \in S_{3}$, where $S_{3}$ is the union of all sets $[x, k, h]$ such that $[x-k+1, k, h]$ belongs to $S_{1}$ given by $(3)$.

We derive Theorem 1 from the following sharpening of Theorem A.

THEOREM 3. Under the assumptions of Theorem A, we have

$$
\mu \leq k-\left[\frac{3}{4} \pi(k)\right]+1-\delta(k)
$$


unless $(x, k) \in S_{3} \cup S_{4}$ where $S_{3}$ is given by Theorem $\mathrm{A}$ and $S_{4}$ is the union of all sets $[x, k, h]$ such that $[x-k+1, k, h]$ belongs to $S_{2}$ given by $(7)$.

We observe that Theorem 1 follows from Theorem 3 by putting $n=$ $x-k+1$ and by choosing $x-f_{1}, x-f_{2}, \ldots, x-f_{\mu}$ as all the factors of $\Delta$ not divisible by a prime greater than $k$, which implies $\omega(\Delta) \geq \pi(k)+k-\mu$.

Further we notice that Theorems $\mathrm{A}$ and 3 are identical when $k \leq 18$. Therefore we shall assume $k \geq 19$ in the proof of Theorem 3 .

The improvement in Theorem 3 depends on sharp estimates of Dusart on $\pi(\nu)$ (see Lemma 1). These have been applied to count the number of terms in $\Delta^{\prime}$ which are primes and the number of terms of the form ap with $2 \leq a \leq 6$ and $p>k$. The latter contribution is crucial for keeping the estimates well under computational range. This is a new feature in the proof of Theorem 3 and it replaces the computational ideas of the proof of (2). It has been applied in the interval $2 k \leq x<7 k$. In fact this interval has been partitioned into several subintervals and the method has been applied to each of those subintervals. This leads to sharper estimates (see Lemmas 5 , $6,8)$. For covering the range $x \geq 7 k$, the ideas of Erdős [5] have been applied (see Lemmas 3, 4, 7). However the estimates of Dusart are not necessary for the improvement given by Corollary 2. In fact the earlier estimates of Rosser and Schoenfeld [9, Theorems 1(3.2), 2(3.3)] of $\pi(\nu)$ suffice.

Theorem 2 follows from the following analogue of Theorem 3 for $x>$ $\frac{29}{12} k-1$.

Theorem 4. Let $x>\frac{29}{12} k-1$ be such that $(x, k) \neq(9,4)$ and $f_{1}<\cdots$ $<f_{\mu}$ be integers in $[0, k)$ satisfying $(15)$. Then

$$
\mu \leq k-\pi(2 k)+\pi(k) .
$$

As in Theorem 2, we observe that the assumptions $x>\frac{29}{12} k-1$ and $(x, k) \neq(9,4)$ in Theorem 4 are necessary. Further we notice that Theorem 4 follows from Theorem A when $k \leq 9$ and therefore we shall assume $k \geq 10$ in the proof of Theorem 4 .

We shall follow the notation of this section throughout the paper. We use MATHEMATICA for the computations in the proofs of the theorems. We thank the referee for his comments on an earlier version of this paper.

2. Proofs of Theorems 3 and 4. We shall assume $k \geq 10$ as stated in Section $1, x \geq 2 k$ and (15) throughout this section and we shall use it without reference. Let

$$
M(k)= \begin{cases}\pi(2 k)-\pi(k) & \text { for } 10 \leq k \leq 18 \\ \max \left(\pi(2 k)-\pi(k),\left[\frac{3}{4} \pi(k)\right]-1\right) & \text { for } k \geq 19\end{cases}
$$


We observe from (5) that

$$
M(k)=\pi(2 k)-\pi(k) \quad \text { for } k \geq 294 .
$$

We begin with the estimates from prime number theory.

LEMMA 1. For $\nu>1$, we have

(i) $\pi(\nu) \leq \frac{\nu}{\log \nu}\left(1+\frac{1.2762}{\log \nu}\right)=: a(\nu)$,

(ii) $\pi(\nu) \geq \frac{\nu}{\log \nu-1}=: b(\nu)$ for $\nu \geq 5393$.

The estimates (i) and (ii) are due to Dusart [2, p. 14]. See also [3, p. 55], [4, p. 414].

The next lemma is Stirling's formula (see [8]).

LEMMA 2. For a positive integer $\nu$, we have

$$
\sqrt{2 \pi \nu} e^{-\nu} \nu^{\nu}<\nu !<\sqrt{2 \pi \nu} e^{-\nu} \nu^{\nu} e^{1 / 12 \nu} .
$$

Lemma 3. Let $x<k^{3 / 2}$. Assume that (15) holds. Then

$$
\left(\begin{array}{l}
x \\
k
\end{array}\right) \leq(2.83)^{k+\sqrt{x}} x^{k-\mu} .
$$

Lemma 3 with $\mu=k$ follows from Erdős [5] and from formula (3.35) of [9] which is $\prod_{p^{m} \leq x} p^{m} \leq(2.83)^{x}$. The proof is similar to that of $[10$, Lemma 3].

Lemma 4. Assume

$$
\mu \geq k-M(k)+1
$$

where $M(k)$ is given by (18). Then

(i) $x<k^{3 / 2}$ for $k \geq 71$,

(ii) $x<k^{7 / 4}$ for $k \geq 25$,

(iii) $x<k^{2}$ for $k \geq 13$,

(iv) $x<k^{9 / 4}$ for $k \geq 10$.

Proof. Let $p^{a} \|\left(\begin{array}{l}x \\ k\end{array}\right)$. We observe that

$$
a=\sum_{\nu=1}^{\infty}\left(\left[\frac{x}{p^{\nu}}\right]-\left[\frac{x-k}{p^{\nu}}\right]-\left[\frac{k}{p^{\nu}}\right]\right) .
$$

Each summand is at most 1 if $p^{\nu} \leq x$ and 0 otherwise. Therefore $a \leq s$ where $p^{s} \leq x<p^{s+1}$. Thus

$$
p^{\operatorname{ord}_{p}\left(\begin{array}{l}
x \\
k
\end{array}\right)}=p^{a} \leq x .
$$


Since $\left(x-f_{1}\right) \cdots\left(x-f_{\mu}\right)$ divides $\left(\begin{array}{l}x \\ k\end{array}\right) k$ !, we observe from (15) that

$$
\left(x-f_{1}\right) \cdots\left(x-f_{\mu}\right) \leq\left(\prod_{p \leq k} p^{\operatorname{ord}_{p}\left(\begin{array}{l}
x \\
k
\end{array}\right)}\right) k ! \leq\left(\prod_{p \leq k} x\right) k !=x^{\pi(k)} k ! .
$$

Also

$$
\left(x-f_{1}\right) \cdots\left(x-f_{\mu}\right) \geq\left(x-f_{\mu}\right)^{\mu} \geq(x-k+1)^{\mu}>x^{\mu}\left(1-\frac{k}{x}\right)^{\mu} .
$$

Comparing this with (22), we get

$$
k !>x^{\mu-\pi(k)}\left(1-\frac{k}{x}\right)^{\mu}
$$

Let $k \geq 71$. We assume that $x \geq k^{3 / 2}$ and we shall arrive at a contradiction. From (23), we have

$$
k !>k^{\frac{3}{2}(\mu-\pi(k))}\left(1-\frac{1}{\sqrt{k}}\right)^{\mu}
$$

and since $\mu \leq k$,

$$
k !>k^{\frac{3}{2}(\mu-\pi(k))}\left(1-\frac{1}{\sqrt{k}}\right)^{k} .
$$

We use (25), (20), (19) and Lemmas 1 (i) and 2 to derive for $k \geq 294$ that

$$
1>2.718 k^{\frac{1}{2}-\frac{3}{\log 2 k}\left(1+\frac{1.2762}{\log 2 k}\right)}\left(1-\frac{1}{\sqrt{k}}\right)
$$

since $\exp \left(\frac{\log 0.3989 k}{k}-\frac{1}{12 k^{2}}\right) \geq 1$. The right hand side of above inequality is an increasing function of $k$ and the inequality is not valid at $k=294$. Thus $k \leq 293$. Further we check that $(25)$ is not valid for $71 \leq k \leq 293$ except at $k=71,73$ by using (20) with $\mu=k-M(k)+1$ and the exact values of $M(k)$ and $k$ !. Let $k=71,73$. We check that (24) is not satisfied if (20) holds with equality sign. Thus we may suppose that (20) holds with strict inequality. Then we find that (25) does not hold. This proves (i). For the proofs of (ii), (iii) and (iv), we may assume that $x \geq k^{7 / 4}$ for $25 \leq k \leq 70$, $x \geq k^{2}$ for $13 \leq k \leq 24$ and $x \geq k^{9 / 4}$ for $k=10,11,12$ respectively and arrive at a contradiction using similar arguments.

Lemma 5. (a) Let $k \geq 19$ and $2 k \leq x<3 k$. Then (16) holds unless $(x, k) \in S_{4}$.

(b) If $\frac{29}{12} k-1<x<3 k$, then (17) holds.

Proof. (a) Let $2 k \leq x<3 k$ with $(x, k) \notin S_{4}$. Then we show that

$$
W\left(\Delta^{\prime}\right) \geq\left[\frac{3}{4} \pi(k)\right]-1
$$


which implies (16). We recall that $W\left(\Delta^{\prime}\right)$ is the number of terms in $\Delta^{\prime}$ divisible by a prime exceeding $k$. Let $2 k+t_{1} k \leq x<2 k+t_{2} k$ with $0 \leq$ $t_{1}<t_{2} \leq 1$ and $t_{2}-t_{1} \leq 1 / 4$. Then $\Delta^{\prime}$ contains a term equal to $p$ for each $x-k<p \leq x$ and a term equal to $2 p$ for each $k<p \leq x / 2$. Therefore

$$
W\left(\Delta^{\prime}\right) \geq \pi(x)-\pi(x-k)+\pi\left(\frac{x}{2}\right)-\pi(k) .
$$

Since $x \geq 2 k+t_{1} k$ and $x-k<k+t_{2} k$, we see from (27) that

$$
W\left(\Delta^{\prime}\right) \geq \pi\left(2 k+t_{1} k\right)-\pi\left(k+t_{2} k\right)+\pi\left(k+t_{1} k / 2\right)-\pi(k) .
$$

Hence it is enough to prove

$$
\begin{aligned}
\pi\left(\left(2+t_{1}\right) k\right)-\pi\left(\left(1+t_{2}\right) k\right)+\pi((1+ & \left.\left.\frac{t_{1}}{2}\right) k\right) \\
& -\pi(k)-\left[\frac{3}{4} \pi(k)\right]+1 \geq 0 .
\end{aligned}
$$

Using Lemma 1(i), (ii) and

$$
\frac{\log Y}{\log Z}=1+\frac{\log (Y / Z)}{\log Z} \quad \text { and } \quad \frac{\log Y}{\log Z-1}=1+\frac{1+\log (Y / Z)}{\log Z-1}
$$

we see that the left hand side of (28) is at least

$$
\begin{aligned}
& \sum_{i=1}^{2} b\left(\frac{2+t_{1}}{i} k\right)-a\left(\left(1+t_{2}\right) k\right)-\frac{7}{4} a(k)+1 \\
& \quad=\frac{k}{\left(\log \left(2+t_{1}\right) k\right)^{2}}\left\{f\left(k, t_{1}, t_{2}\right)-g\left(k, t_{1}, t_{2}\right)-\frac{7}{4} g\left(k, t_{1}, 0\right)\right\}+1
\end{aligned}
$$

for $k \geq 5393$, where

$$
\begin{aligned}
f\left(k, t_{1}, t_{2}\right)= & \left(1.5 t_{1}-t_{2}+\frac{1}{4}\right)\left(\log \left(2+t_{1}\right) k\right) \\
& +\sum_{i=1}^{2} \frac{\left(2+t_{1}\right)(1+\log i)}{i}\left(1+\frac{1+\log i}{\log \left(\left(2+t_{1}\right) k / i\right)-1}\right)
\end{aligned}
$$

and

$$
\begin{aligned}
g\left(k, t_{1}, t_{2}\right)= & \left(1+t_{2}\right)\left(1+\frac{\log \left(\frac{2+t_{1}}{1+t_{2}}\right)}{\log \left(\left(1+t_{2}\right) k\right)}\right) \\
& \times\left(1.2762+\log \left(\frac{2+t_{1}}{1+t_{2}}\right)+\frac{1.2762 \log \left(\frac{2+t_{1}}{1+t_{2}}\right)}{\log \left(\left(1+t_{2}\right) k\right)}\right) .
\end{aligned}
$$

Then we have

$$
k f^{\prime}\left(k, t_{1}, t_{2}\right)=\left(1.5 t_{1}-t_{2}+\frac{1}{4}\right)-\sum_{i=1}^{2}\left(\frac{2+t_{1}}{i}\right)\left(\frac{1+\log i}{\log \left(\left(2+t_{1}\right) k / i\right)-1}\right)^{2} .
$$


We write

$$
1.5 t_{1}-t_{2}+\frac{1}{4}=0.5 t_{1}-\left(t_{2}-t_{1}\right)+\frac{1}{4}
$$

to observe that the left hand side is positive unless $\left(t_{1}, t_{2}\right)=(0,1 / 4)$ and we shall always assume that $\left(t_{1}, t_{2}\right) \neq(0,1 / 4)$.

Let $k_{0}=k_{0}\left(t_{1}, t_{2}\right)$ be such that $k f^{\prime}\left(k, t_{1}, t_{2}\right)$ is positive at $k_{0}$. Since $k f^{\prime}\left(k, t_{1}, t_{2}\right)$ is an increasing function of $k$, we see that $f\left(k, t_{1}, t_{2}\right)$ is also an increasing function of $k$ for $k \geq k_{0}$. Also $g\left(k, t_{1}, t_{2}\right)$ is a decreasing function of $k$. Hence (29) is an increasing function of $k$ for $k \geq k_{0}$. Let $k_{1}=k_{1}\left(t_{1}, t_{2}\right)$ $\geq k_{0}$ be such that (29) is non-negative at $k_{1}$. Then (28) is valid for $k \geq k_{1}$. For $k<k_{1}$, we check inequality (28) by using the exact values of $\pi(\nu)$. Again for $k$ not satisfying (28), we take $x=2 k+r$ with $t_{1} k \leq r<t_{2} k$ and check that the right hand side of (27) is at least the right hand side of (26).

Let $2 k \leq x<\frac{49}{24} k$. Then $t_{1}=0, t_{2}=1 / 24$ and we find $k_{1}=5393$ by (29). For $k<5393$, we check that (28) holds except at the following values of $k$ :

$$
\left\{\begin{array}{l}
19,20,47,48,71,72,73,74,80,81,83,86,89,103,104,105,106,107,108, \\
109,110,111,112,113,114,115,116,134,151,152,153,167,168,172, \\
173,174,175,176,186,191,192,193,194,195,196,197,198,199,200,203, \\
204,266,267,268,269,270,271,272,273,274,275,276,277,278,279,280, \\
281,282,283,284,285,286,287,288,293,295,296,299,449,450,451,452, \\
453,463,464,467,468,469,470,472,473,480,481,482,483,484,485,491, \\
503,504,505,506,635,636,637,638,683,704,709,710,711,712,713,714 .
\end{array}\right.
$$

For the above values of $k$, we write $x=2 k+r$ with $0 \leq r<k / 24$ and show that the right hand side of (27) is at least the right hand side of (26) since $(x, k) \notin S_{4}$.

We apply similar arguments to the intervals

$$
\left\{\begin{array}{l}
\frac{49}{24} k \leq x<\frac{25}{12} k, \frac{25}{12} k \leq x<\frac{13}{6} k, \frac{13}{6} k \leq x<\frac{9}{4} k, \frac{9}{4} k \leq x<\frac{19}{8} k, \\
\frac{19}{8} k \leq x<\frac{5}{2} k, \frac{5}{2} k \leq x<\frac{11}{4} k, \frac{11}{4} k \leq x<3 k .
\end{array}\right.
$$

We find $k_{1}=5393$ for each of these intervals. Further the contributions of $\pi\left(\left(1+t_{1} / 2\right) k\right)-\pi(k)$ in $(28)$ and $\pi(x / 2)-\pi(k)$ in (27) are necessary for the proof. Finally, we observe that (26) is valid for all pairs $(x, k)$ with $x \geq \frac{19}{8} k$. This proves (a).

(b) We divide $\frac{29}{12} k-1<x<3 k$ into the subintervals $\frac{29}{12} k-1<x<\frac{5}{2} k$, $\frac{5}{2} k \leq x<\frac{21}{8} k, \frac{21}{8} k \leq x<\frac{11}{4} k, \frac{11}{4} k \leq x<3 k$. We apply the arguments of (a) to each of these subintervals to conclude that $W\left(\Delta^{\prime}\right) \geq \pi(2 k)-\pi(k)$, which implies (17). 
In view of Lemma 5 , it remains to prove Theorems 3 and 4 for $x \geq 3 k$, which we assume. Further we may also suppose (20). Otherwise both (16) with $k \geq 19$ and (17) follow. Now we derive from Lemma 4 that $x<k^{9 / 4}$. On the other hand, we prove $x \geq k^{9 / 4}$. This is a contradiction. We split the proof of $x \geq k^{9 / 4}$ in the following three lemmas.

Lemma 6. If (20) holds and $x \geq 3 k$, then $x \geq 7 k$.

LEMMA 7. If (20) holds and $x \geq 7 k$, then $x \geq k^{3 / 2}$.

LEMMA 8. If (20) holds and $x \geq k^{3 / 2}$, then $x \geq k^{9 / 4}$.

We shall give proofs of Lemmas 6-8 in the next section; then the proofs of Theorems 3 and 4 will be complete.

\section{Proofs of Lemmas 6-8}

Proof of Lemma 6. Suppose $3 k \leq x<7 k$. We show that

$$
W\left(\Delta^{\prime}\right) \geq M(k),
$$

which contradicts (20). Let $\left(s+t_{1}\right) k \leq x<\left(s+t_{2}\right) k$ with integers $3 \leq s \leq 6$ and $t_{1}, t_{2} \in\{0,1 / 4,1 / 2,3 / 4,1\}$ such that $t_{2}-t_{1}=1 / 4$. Then $\Delta^{\prime}$ contains a term equal to $i p$ with $(x-k) / i<p \leq x / i$ for each $i$ with $1 \leq i<s$ and a term equal to $s p$ for $k<p \leq x / s$. Therefore

$$
W\left(\Delta^{\prime}\right) \geq \sum_{i=1}^{s-1}\left(\pi\left(\frac{x}{i}\right)-\pi\left(\frac{x-k}{i}\right)\right)+\pi\left(\frac{x}{s}\right)-\pi(k) .
$$

Since $x \geq\left(s+t_{1}\right) k$ and $x-k<\left(s-1+t_{2}\right) k$, we observe from (31) that

$$
W\left(\Delta^{\prime}\right) \geq \sum_{i=1}^{s-1}\left(\pi\left(\frac{s+t_{1}}{i} k\right)-\pi\left(\frac{s-1+t_{2}}{i} k\right)\right)+\pi\left(\frac{s+t_{1}}{s} k\right)-\pi(k) .
$$

Hence it is enough to show

$$
\begin{aligned}
\sum_{i=1}^{s-1}\left(\pi\left(\frac{s+t_{1}}{i} k\right)-\pi\left(\frac{s-1+t_{2}}{i} k\right)\right)+\pi & \left(\frac{s+t_{1}}{s} k\right) \\
& -\pi(k)-M(k) \geq 0 .
\end{aligned}
$$

Using (19) and Lemma 1(i), (ii), we see that the left hand side of (32) is at least

$$
\sum_{i=1}^{s-1}\left(b\left(\frac{s+t_{1}}{i} k\right)-a\left(\frac{s-1+t_{2}}{i} k\right)\right)+b\left(\frac{s+t_{1}}{s} k\right)-a(2 k)
$$

$$
=\frac{k}{\left(\log \left(s+t_{1}\right) k\right)^{2}}\left\{F\left(k, s, t_{1}, t_{2}\right)-\sum_{i=1}^{s-1} G\left(k, s, t_{1}, t_{2}, i\right)-G\left(k, s, t_{1}, 1, s / 2\right)\right\}
$$


for $k \geq 5393$, where

$$
\begin{aligned}
F\left(k, s, t_{1}, t_{2}\right)= & \left(\sum_{i=1}^{s-1}\left(\frac{1+t_{1}-t_{2}}{i}\right)+\frac{t_{1}}{s}-1\right)\left(\log \left(s+t_{1}\right) k\right) \\
& +\sum_{i=1}^{s} \frac{\left(s+t_{1}\right)(1+\log i)}{i}\left(1+\frac{1+\log i}{\log \left(\left(s+t_{1}\right) k / i\right)-1}\right)
\end{aligned}
$$

and

$$
\begin{aligned}
G\left(k, s, t_{1}, t_{2}, i\right)= & \left(\frac{s-1+t_{2}}{i}\right)\left(1+\frac{\log \left(\frac{\left(s+t_{1}\right) i}{s-1+t_{2}}\right)}{\log \left(\frac{s-1+t_{2}}{i} k\right)}\right) \\
& \times\left(1.2762+\log \left(\frac{\left(s+t_{1}\right) i}{s-1+t_{2}}\right)+\frac{1.2762 \log \left(\frac{\left(s+t_{1}\right) i}{s-1+t_{2}}\right)}{\log \left(\frac{s-1+t_{2}}{i} k\right)}\right) .
\end{aligned}
$$

Then

$$
\begin{aligned}
k F^{\prime}\left(k, s, t_{1}, t_{2}\right)= & \left(\sum_{i=1}^{s-1}\left(\frac{1+t_{1}-t_{2}}{i}\right)+\frac{t_{1}}{s}-1\right) \\
& -\sum_{i=1}^{s} \frac{s+t_{1}}{i}\left(\frac{1+\log i}{\log \left(\left(s+t_{1}\right) k / i\right)-1}\right)^{2} .
\end{aligned}
$$

If $s=2$, we note that $F$ and $G$ are functions similar to $f$ and $g$ appearing in Lemma 5. As in Lemma 5 , we find $K_{1}:=K_{1}\left(s, t_{1}, t_{2}\right)$ such that (33) is non-negative at $k=K_{1}$ and it is increasing for $k \geq K_{1}$. Hence (32) is valid for $k \geq K_{1}$. For $k<K_{1}$, we check inequality (32) by using the exact values of $\pi(\nu)$. Again for $k$ not satisfying (32), we take $x=s k+r$ with $t_{1} k \leq r<t_{2} k$ and check that the right hand side of (31) is at least the right hand side of (30).

Let $3 k \leq x<\frac{13}{4} k$. Here $t_{1}=0, t_{2}=\frac{1}{4} k$ and we find $K_{1}=29000$. We check that (32) holds for $3 \leq k<29000$ except at $k=10,12,19,22,40,42$, $52,55,57,100,101,102,124,125,126,127,142,143$. For these values of $k$, putting $x=3 k+r$ with $0 \leq r<\frac{1}{4} k$, we show that the right hand side of (31) is at least the right hand side of (30). Hence the assertion follows in $3 k \leq x<\frac{13}{4} k$.

For $x \geq \frac{13}{4} k$, we apply similar arguments to the intervals $\left(s+t_{1}\right) k \leq$ $x<\left(s+t_{2}\right) k$ with integers $3 \leq s \leq 6$ and $t_{1}, t_{2} \in\{0,1 / 4,1 / 2,3 / 4,1\}$ such that $t_{2}-t_{1}=1 / 4$. We find $K_{1}=5393$ for each of these intervals except for $6 k \leq x<\frac{25}{4} k$ where $K_{1}=5500$.

Proof of Lemma \%. We argue by contradiction. We assume (20) and $7 k \leq x<k^{3 / 2}$. Then $k \geq 50$. Further by Lemma 3 and $\left(\begin{array}{l}x \\ k\end{array}\right) \geq\left(\begin{array}{c}7 k \\ k\end{array}\right)$, we have 


$$
\left(\begin{array}{c}
7 k \\
k
\end{array}\right)<(2.83)^{k+k^{3 / 4}} k^{\frac{3}{2}(M(k)-1)}
$$

since $x<k^{3 / 2}$. We observe from Lemma 2 that

$$
\begin{aligned}
\left(\begin{array}{c}
7 k \\
k
\end{array}\right)=\frac{(7 k) !}{k !(6 k) !} & >\frac{\sqrt{2 \pi 7 k} e^{-7 k}(7 k)^{7 k}}{\sqrt{2 \pi k} e^{-k} k^{k} e^{1 / 12 k} \sqrt{2 \pi 6 k} e^{-6 k}(6 k)^{6 k} e^{1 / 72 k}} \\
& >\frac{0.4309}{\sqrt{k}} e^{-7 / 72 k}(17.65)^{k}
\end{aligned}
$$

Combining this with (34), we get

$$
1>\exp \left(\log (0.4309 k)-\frac{7}{72 k}\right)(17.65)^{k}(2.83)^{-k-k^{3 / 4}} k^{-\frac{3}{2} M(k)} .
$$

Using (19), Lemma 1(i), (ii) and $\exp \left(\frac{\log (0.4309 k)}{k}-\frac{7}{72 k^{2}}\right) \geq 1$, we derive for $k \geq 5393$ that

$$
\begin{aligned}
1 & >6.2367(2.83)^{-k^{-1 / 4}} k^{-\frac{3}{\log 2 k}\left(1+\frac{1.2762}{\log 2 k}\right)+\frac{3}{2(\log k-1)}} \\
& >6.2367 \exp \left(\frac{3}{2}+\frac{3}{2 \log k-2}\right)(2.83)^{-k^{-1 / 4}} k^{-\frac{3}{\log 2 k}\left(1+\frac{1.2762}{\log 2 k}\right)} \\
& >27.95(2.83)^{-k^{-1 / 4}} k^{-\frac{3}{\log 2 k}\left(1+\frac{1.2762}{\log 2 k}\right)}=: h(k),
\end{aligned}
$$

since $\exp \left(\frac{3}{2 \log k-2}\right)>1$ for $k \geq 3$. We see that $h(k)$ is an increasing function of $k$ and $h(k)>1$ at $k=5393$. Therefore $k<5393$. By using the exact values of $M(k)$, we now check that (35) does not hold for $50 \leq k<5393$.

Proof of Lemma 8. We argue by contradiction. Assume (20) and $k^{3 / 2} \leq$ $x<k^{9 / 4}$. We derive from Lemma 4 that $k \leq 70$. Let $k=10,11,12,13$. By Lemmas 4, 6 and 7 , we can take $\max \left(7 k, k^{3 / 2}\right) \leq x<k^{9 / 4}$ for $k=10,11,12$ and $\max \left(7 k, k^{3 / 2}\right) \leq x<k^{2}$ for $k=13$. For these values of $x$ and $k$, we find that

$$
W\left(\Delta^{\prime}\right) \geq \sum_{i=1}^{6}\left(\pi\left(\frac{x}{i}\right)-\pi\left(\frac{x-k}{i}\right)\right) \geq M(k),
$$

which contradicts (20).

Therefore we assume $k \geq 14$. Let $k^{3 / 2} \leq x<k^{25 / 16}$. By Lemmas 6 and 7 , we can take $x \geq \max \left(7 k, k^{3 / 2}\right)$ so that we can assume $k \geq 32$. Then

$$
\left(\begin{array}{l}
x \\
k
\end{array}\right) \geq\left(\begin{array}{c}
\max \left(7 k,\left\{k^{3 / 2}\right\}\right) \\
k
\end{array}\right)
$$

where $\{\nu\}$ denotes the least integer $\geq \nu$. From (21), we have

$$
\operatorname{ord}_{p}\left(\left(\begin{array}{l}
x \\
k
\end{array}\right)\right) \leq\left[\frac{\log x}{\log p}\right] \leq\left[\frac{25}{16} \frac{\log k}{\log p}\right]
$$


and hence

$$
\left(\begin{array}{l}
x \\
k
\end{array}\right) \leq\left(\prod_{i=1}^{\pi(k)} p_{i}^{\left[\frac{25}{16} \frac{\log k}{\log p_{i}}\right]}\right) x^{k-\mu}<\left(\prod_{i=1}^{\pi(k)} p_{i}^{\left[\frac{25}{16} \frac{\log k}{\log p_{i}}\right]}\right) k^{\frac{25}{16}(M(k)-1)}
$$

by $(20)$. Combining the above estimates for $\left(\begin{array}{l}x \\ k\end{array}\right)$, we get

$$
\left(\begin{array}{c}
\max \left(7 k,\left\{k^{3 / 2}\right\}\right) \\
k
\end{array}\right)<\left(\prod_{i=1}^{\pi(k)} p_{i}^{\left[\frac{25}{16} \frac{\log k}{\log p_{i}}\right]}\right) k^{\frac{25}{16}(M(k)-1)},
$$

which is not possible for $32 \leq k \leq 70$. By similar arguments, we arrive at a contradiction for $\max \left(7 \bar{k}, k^{25 / 16}\right) \leq x<k^{26 / 16}$ in $23 \leq k \leq 70$, $\max \left(7 k, k^{26 / 16}\right) \leq x<k^{27 / 16}$ in $17 \leq k \leq 70$ and $\max \left(7 k, k^{27 / 16}\right) \leq x<k^{7 / 4}$ in $14 \leq k \leq 70$ except at $k=16$. Let $k=16$ and $\max \left(7 k, k^{27 / 16}\right) \leq x<k^{7 / 4}$. Then we observe that

$$
W\left(\Delta^{\prime}\right) \geq \sum_{i=1}^{6}\left(\pi\left(\frac{x}{i}\right)-\pi\left(\frac{x-16}{i}\right)\right) \geq 5=M(16)
$$

which contradicts $(20)$.

Now we consider $x \geq k^{7 / 4}$. We observe that $k^{7 / 4} \geq 7 k$ since $k \geq 14$. Further we derive from Lemma 4 that $k \leq 24$. We apply similar arguments for $14 \leq k \leq 24$ as above to arrive at a contradiction in the intervals $k^{7 / 4} \leq x<k^{15 / 8}$ except at $k=16, k^{15 / 8} \leq x<k^{31 / 16}$ and $k^{31 / 16} \leq x<k^{2}$. The case $k=16$ and $k^{7 / 4} \leq x<k^{15 / 8}$ is excluded similarly to the above.

\section{References}

[1] E. Catalan, Note extraite d'une lettre adressée à l'éditeur, J. Reine Angew. Math. 27 (1844), 1992.

[2] P. Dusart, Autour de la fonction qui compte le nombre de nombres premiers, Ph.D. thesis, Université de Limoges, 1998.

[3] - Inégalités explicites pour $\psi(X), \theta(X), \pi(X)$ et les nombres premiers, C. R. Math. Rep. Acad. Sci. Canada 21 (1999), 53-59.

[4] - The kth prime is greater than $k(\log k+\log \log k-1)$ for $k \geq 2$, Math. Comp. 68 (1999), 411-415.

[5] P. Erdős, A theorem of Sylvester and Schur, J. London Math. Soc. 9 (1934), 282-288.

[6] P. Mihăilescu, Primary cyclotomic units and a proof of Catalan's conjecture, submitted.

[7] A. Mukhopadhyay and T. N. Shorey, Square free part of products of consecutive integers, Publ. Math. Debrecen 64 (2004), 79-99.

[8] H. Robbins, A remark on Stirling's formula, Amer. Math. Monthly 62 (1955), 26-29.

[9] J. B. Rosser and L. Schoenfeld, Approximate formulas for some functions of prime numbers, Illinois J. Math. 6 (1962), 64-94.

[10] N. Saradha and T. N. Shorey, Almost squares and factorisations in consecutive integers, Compositio Math. 138 (2003), 113-124. 
[11] N. Saradha and T. N. Shorey, Almost squares in arithmetic progression, ibid. 138 (2003), 73-111.

[12] J. J. Sylvester, On arithmetical series, Messenger of Mathematics 21 (1892), 1-19, 87-120, and Mathematical Papers, 4 (1912), 687-731.

[13] R. Tijdeman, On the equation of Catalan, Acta Arith. 29 (1976), 197-209.

School of Mathematics

Tata Institute of Fundamental Research

Homi Bhabha Road

Mumbai 400 005, India

E-mail: shanta@math.tifr.res.in shorey@math.tifr.res.in

Received on 16.4.2003

and in revised form on 19.1.2004 